\title{
Preventing 'SIRTain' death by mutant huntingtin
}

Huntington's disease is an inherited neurodegenerative disease caused by a glutamine repeat expansion in huntingtin (HTT) protein that results in HTT accumulation. Studies have shown that calorie restriction ameliorates the pathogenesis of the disease in mice, but the role of the NAD-dependent protein deacetylase sirtuin 1 (SIRT1), which is one of the mediators of the beneficial roles of calorie restriction in Huntington's disease, was unclear. Two studies in Nature Medicine reveal a neuroprotective role for this enzyme and, thus, potential new therapeutic opportunities for treating the disease.

Duan and colleagues crossed Sirt1-transgenic mice, in which the enzyme is highly expressed in the brain, with mice expressing an aminoterminal mutant HTT fragment (N171-82Q) - a model for Huntington's disease. They found that the overexpression of Sirt1 delayed the onset and progression of the motor deficits that characterize the N171-82Q mice. In addition, increased levels of SIRT1 reduced the magnitude of atrophy in the striatum and neocortex without causing changes in the levels of mutant HTT. Overexpression of the enzyme had similar effects in mice expressing full-length mutant HTT, another mouse model of Huntington's disease, thus confirming its neuroprotective role.

The paper by Krainc and colleagues also showed that increased expression of Sirt1 in another Huntington's disease model (R6/2 mice) protected against striatal degeneration. It even extended the survival of male R6/2 mice; this gender-specific effect might be due to the higher expression of Sirt 1 in the female transgenic mice compared with males. Conversely, the knockout of brain-specific Sirt 1 accelerated the decrease in striatal volume and onset of motor deficits that are typically observed in R6/2 mice. Unlike the study by Duan's group, Krainc and colleagues observed a decrease in mutant HTT aggregates in mice overexpressing Sirt 1 - a discrepancy that might be explained by differences between the mouse models used in the two studies.

Further experiments by Duan and colleagues in PC12 cells showed that the deacetylase activity of SIRT1 was required to protect these cells from mutant HTT-induced toxicity. Interestingly, the levels of acetylated forkhead box protein O3A (FOXO3A), a SIRT1 substrate, were increased in cells expressing mutant HTT, suggesting that mutant HTT inhibits the activity of SIRT1. The levels of other known SIRT1 targets: brain-derived neurotrophic factor (BDNF) and dopamine- and cyclic AMP-regulated neuronal phosphoprotein 32 (DARPP32; also known as PPP1R1B), which are decreased in the Huntington's disease mouse models, were restored in the mice overexpressing Sirt1. These findings led the authors to conclude that HTT-mediated inhibition of SIRT1 activity is a key mechanism underlying the neuropathology of the disease.

Krainc and colleagues shed further light on the molecular mechanism through which the deacetylase activity of SIRT1 protects neurons from mutant HTT. They showed that, in cell cultures, SIRT1 deacetylates and thereby activates CAMP-responsive element-binding protein 1 (CREB)-regulated transcription coactivator 1 (TORC1), a previously unknown SIRT1 substrate, leading to an increase in CREB-mediated transcription of BDNF. Importantly, the interaction of mutant HTT with SIRT1 prevented the activation of TORC1 and its interaction with CREB. These findings indicate that the TORC1-CREB pathway is an important mediator of the protective effects of SIRT1 in mutant HTT-expressing neurons. Moreover, as TORC1 is only expressed in the CNS and testis, its activation could be crucial to the neuronal function of SIRT1.

The two papers highlight a new role for SIRT1 in Huntington's disease and suggest that SIRT1-targeted therapies may offer new treatment opportunities.

Monica Hoyos Flight

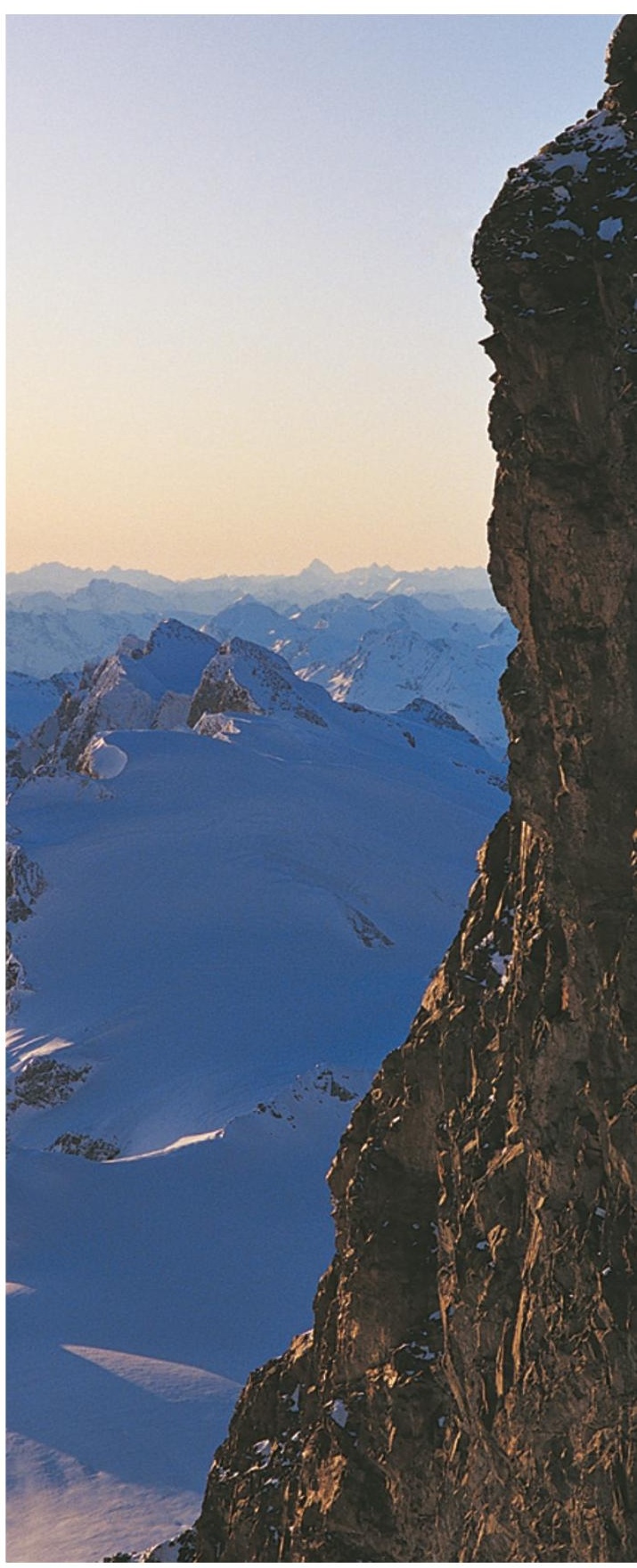

ORIGINAL RESEARCH PAPERS Jiang, M. et al. Neuroprotective role of Sirt1 in mammalian models of Huntington's disease through activation of multiple Sirt1 targets. Nature Med. 18, 153-158 (2012) | Jeong, H. et al. Sirt1 mediates neuroprotection from mutant huntingtin by activation of the TORC1 and CREB transcriptional pathway. Nature Med. 18, 159-165 (2012) 